\title{
National Security, Forensics and Mobile Communications
}

\author{
David Naccache \\ Ecole Normale Superieure, France \\ david.naccache@ens.fr
}

\begin{abstract}
There are nearly 1.5 billion handset users in the world. As the recent attacks in London illustrate, this proliferation of inexpensive mobile phones provides criminals and terrorists with flexible communication means. In this talk we will describe the forensic methodology used for analysing SIM cards and handsets within the French legal context. We will describe specific technical problems applicable to the analysis of cards (e.g., the impossibility to interact with a SIM without altering its internal state), underline seizure details, demonstrate the tools allowing to extract the contents of a handset and a SIM knowing their associated PIN codes. We will overview some of the "heavy" analysis methods (physical reverse-engineering) used with various degrees of success when PIN codes are unknown and describe the protocol allowing to negotiate with the judiciary authorities an evidence destruction risk before undertaking the actual analysis of the SIMs and handsets.
\end{abstract}

